\title{
INFINITE ERGODIC INDEX OF THE EHRENFEST WIND-TREE MODEL
}

\author{
ALBA MÁLAGA SABOGAL AND SERGE TROUBETZKOY
}

\begin{abstract}
The set of all possible configurations of the Ehrenfest wind-tree model endowed with the Hausdorff topology is a compact metric space. For a typical configuration we show that the windtree dynamics has infinite ergodic index in almost every direction. In particular some ergodic theorems can be applied to show that if we start with a large number of initially parallel particles their directions decorrelate as the dynamics evolve answering the question posed by the Ehrenfests.
\end{abstract}

\section{INTRODUCTION}

In 1912 Paul and Tatiana Ehrenfest wrote a seminal article on the foundations of Statistical Mechanics in which the wind-tree model was introduced in order to interpret the work of Boltzmann and Maxwell on gas dynamics [EhEh]. In the wind-tree model a point particle moves without friction on the plane with infinitely many rigid obstacles removed, and collides elastically with the obstacles. The Ehrenfests' paper dates from times when the notions of probability theory where not yet rigorously defined. Thus they could not describe the distribution of the obstacles in a probabilistic way, they used the word "irregular" to describe it. However, they made precise what they did expected from the placement of the obstacles: obstacles are identical squares, all parallel to each other, the placement is irregular, every portion of the plane contains about the same number of obstacles, and the distances between the obstacles are large in comparison to the obstacle's size.

If we fix the direction of the particle, the billiard flow will take only four directions. The Ehrenfests asked the following question: start $K$ particles in a given direction, will the number of particles in each of the four directions asymptotically equalize to about $K / 4$ ? To answer this question we study the ergodic properties of the wind-tree model.

We thank Jack Milnor for suggesting a nice presentation of our topology. AMS acknowledges that this work was started during a post-doc funded by the A*MIDEX project (ANR-11-IDEX-0001-02), funded itself by the "Investissements d'avenir" program of the French Government, managed by the French National Research Agency (ANR)". She continued working on this project during the ATER position she held at Mathematics Laboratory in Orsay in 2015-2016. ST gratefully acknowledges the support of project APEX "Systèmes dynamiques: Probabilités et Approximation Diophantienne PAD" funded by the Région PACA. 
Interestingly the birth of ergodic theory can be traced back to the Ehrenfests' article in which the word ergodic was used for the first time with a close mathematical meaning to the current one GaBoGe. We consider the set of all possible configurations and introduce a canonical topology which makes it a compact metric space. We show that for (Baire) generic configurations, for almost every direction the billiard flow has infinite ergodic index, i.e., all its powers are ergodic. In a finite measure space this would be equivalent to saying that the flow is weakly mixing. The asymptotic equalization of the directions of $K$ particles in several senses then follows from various ergodic theorems (note that we are in the framework of infinite ergodic theory here, so the Birkhoff ergodic theorem is not directly applicable).

In two previous articles we have considered a subset of configurations which are small perturbations of lattice configurations, and we showed that the generic wind-tree is minimal and ergodic in almost every direction [MSTr1, MSTr2]. The topology considered in these two articles is equivalent to the one considered in this article. Furthermore the proofs of these two results hold mutatis mutandis in the more general setting which we consider here.

There have been a number of results on the wind-tree model DeCoVB, $\mathrm{Ga}$, $\mathrm{HaCo}, \mathrm{HaCo1}$, Tr, VBHa, WoLa, and on the wind-tree model with periodical distribution of obstacles of squares, rectangles and more recently other polygonal shapes [AvHu, BaKhMaPl, BiRo, De, DeHuLe, DeZo, FrHu, FrUl, HaWe, HuLeTr.

\section{Definitions AND MAIN RESUltS}

For sake of simplicity, a square whose sides are parallel to lines $y=$ $\pm x$ will be referred to as rhombus in the rest of the article. The $\mathcal{L}_{1}$ distance in $\mathbb{R}^{2}$ will be denoted by $d$. Note that balls with respect to this distance are rhombii.

Fix $s>0$. A configuration is an at most countable collection of rhombii with diameter $s$, whose interiors are pairwise disjoint. Since $s$ is fixed it is enough to note the centers of the rhombii, thus a configuration $g$ is an at most countable subset of $\mathbb{R}^{2}$ such that if $z_{1}, z_{2} \in g$ then $d\left(z_{1}, z_{2}\right) \geq s$.

To define a topology on the set of configurations consider polar coordinates $(r, \theta)$ on the plane. Each point $(r, \theta)$ in the plane is the stereographic projection of a point in the sphere with spherical coordinates $(2 \arctan (1 / r), \theta)$. Apply the inverse of the stereographic projection to a configuration $g$ to obtain a subset of the sphere. Let $\hat{g}$ denote the union of this set with the north pole of the sphere denoted by $\{\infty\}$, it is a closed subset of the sphere. The topology we define on the set of configurations is then induced by the Hausdorff distance $d_{H}$ given by

$$
d_{H}\left(g_{1}, g_{2}\right)=\max \left(\sup _{z_{1} \in \hat{g}_{1}} \inf _{z_{2} \in \hat{g}_{2}} \rho\left(z_{1}, z_{2}\right), \sup _{z_{2} \in \hat{g}_{2}} \inf _{z_{1} \in \hat{g}_{1}} \rho\left(z_{1}, z_{2}\right)\right) \text {. }
$$


Here $\rho$ denotes the geodesic distance on the sphere, i.e., the length of the shortest path from one point to another along the great circle passing through them. Let Conf be the set of all configurations.

Proposition 1. (Conf, $\left.d_{H}\right)$ is compact metric space, thus a Baire space.

The proposition is proven in the appendix. Let $\mathcal{U}_{\varepsilon}(g)$ be the set of all configurations that are at most $\varepsilon$-close to $g$

$$
\mathcal{U}_{\varepsilon}(g):=\left\{g^{\prime} \mid d_{H}\left(g^{\prime}, g\right)<\varepsilon\right\} .
$$

Proposition 2. There is a dense $G_{\delta}$ subset $G$ of $\left(\right.$ Conf, $\left.d_{H}\right)$ such that for each $g \in G$

(1) $g$ is an infinite configuration,

(2) every pair of points $z_{1}, z_{2} \in g$ satisfy $d\left(z_{1}, z_{2}\right)>s$.

Remark. Point (2) means that the obstacles centered at $z_{1}$ and $z_{2}$ do not intersect.

Proof. There are infinite configurations arbitrarily close to any finite configuration, thus we can choose a countable dense set $\left\{g_{n}: n \in \mathbb{N}\right\}$ of infinite configurations. Let $\varepsilon\left(g_{n}\right)>0$ be the infinimum of $\varepsilon$ such that there are at least $n$ distinct points $z \in g_{n}$ satisfying $\rho(z, \infty)>\varepsilon$. Denote this necessarily finite set of points by $B\left(g_{n}\right)$. Clearly

$$
G:=\bigcap_{m=1}^{\infty} \bigcup_{n \geq m} U_{\varepsilon\left(g_{n}\right)}\left(g_{n}\right)
$$

is a dense $G_{\delta}$ set. If $g \in G$, then $g$ is in $U_{\varepsilon\left(g_{n}\right)}\left(g_{n}\right)$ for an arbitrarily large $n$ and thus $g$ is an infinite configuration.

Now additionally suppose that $\left\{g_{n}\right\}$ satisfies

$$
\min \left\{d\left(z_{1}, z_{2}\right): z_{1}, z_{2} \in B\left(g_{n}\right)\right\} \geq s+1 / n .
$$

We also require that $\varepsilon\left(g_{n}\right)$ satisfies : for any $h \in U_{\varepsilon\left(g_{n}\right)}\left(g_{n}\right)$ we have

$$
\min \left\{d\left(z_{1}, z_{2}\right): z_{1}, z_{2} \in B\left(g_{n}\right)\right\} \geq s+1 / 2 n .
$$

Point (2) follows directly.

Fix $g \in$ Conf. The wind-tree table $\mathcal{B}^{g}$ is the plane $\mathbb{R}^{2}$ with the interiors of the union of the trees removed. Fix $\theta \in \mathbb{S}^{1}$. The billiard flow $\phi_{t}^{g, \theta}$ in the direction $\theta$ or simply $\phi_{t}^{\theta}$ is the free motion in the interior of $\mathcal{B}^{g}$ with elastic collision from the boundary of $\mathcal{B}^{g}$ (the boundary of the union of the trees). Once launched in the direction $\theta$, the billiard direction can only achieve four directions $[\theta]:=\{ \pm \theta, \pm(\pi-\theta)\}$; thus the phase space $X^{g, \theta}$ of the billiard flow in the direction $\theta$ is a subset of the cartesian product of $\mathcal{B}^{g}$ with these four directions. We agree that if a billiard orbit hits a corner of a tree, the outcome of the collision is not defined, and the billiard orbit stops there, its future is not defined anymore. Note that in this notation $\phi_{t}^{\theta}, \phi_{t}^{-\theta}, \phi_{t}^{\pi-\theta}$ and $\phi_{t}^{\theta-\pi}$ are all the same. 
A flow $\psi_{t}$ preserving a Borel measure $m$ is called ergodic if for each Borel measurable set $A, m\left(\psi_{t}(A) \triangle A\right)=0 \forall t \in \mathbb{R}$ implies that $m(A)=$ 0 or $m\left(A^{c}\right)=0$. The flow $\psi_{t}$ is said to have infinite ergodic index if for each integer $K \geq 1$ the $K$-fold product flow $\psi_{t} \times \cdots \times \psi_{t}$ is ergodic with respect to the $K$-fold product measure $m \times \cdots \times m$. It is a well known fact that in the finite measure case the notion of infinite ergodic index is equivalent to weak-mixing. However we are working in the context of an infinite measure preserving flow.

For each direction $\theta$, the billiard flow $\phi_{t}^{\theta}$ preserves the area measure $\mu$ on $\mathcal{B}^{g}$ times a discrete measure on $[\theta]$, we will also call this measure $\mu$. Note that $\mu$ is an infinite measure. The billiard flow on the full phase space preserves the volume measure $\mu \times \lambda$ with $\lambda$ the length measure on $\mathbb{S}^{1}$. Let $K \geq 1$, and let $\vec{\theta}=\left(\theta_{1}, \ldots, \theta_{K}\right)$ be a vector of directions. Then we note the product billiard flow $\phi_{t}^{\vec{\theta}}:=\phi_{t}^{\theta_{1}} \times \cdots \times \phi_{t}^{\theta_{K}}$. This flow preserves the measure $\mu^{K}:=\mu \times \cdots \times \mu$.

Now we can state our main result.

Theorem 3. For any $s>0$ there is a dense $G_{\delta}$ subset $G$ of Conf and a dense $G_{\delta}$ set of full measure of directions $\mathcal{H}$, for every integer $K \geq 1$ there is a dense $G_{\delta}$ set $\mathcal{H}(K)$ of full measure of $K$-tuples of directions such that for each $g \in G$

(1) the flow $\phi_{t}^{\theta}$ has infinite ergodic index for every $\theta \in \mathcal{H}$ and

(2) the flow $\phi_{t}^{\vec{\theta}}$ is ergodic for every $\vec{\theta} \in \mathcal{H}(K)$.

Remark. We do not know that the set $\mathcal{H}(K)$ has product structure, thus (1) does not follow from (2).

2.1. The precise question posed by the Ehrenfests. Consider a large but finite number $K$ of initial points in the wind-tree model in a given direction $\theta$. The Ehrenfests asked do the particles directions asymptotically equalize under the wind-tree dynamics, i.e., are there approximately $K / 4$ particles in each direction after a large time.

This question is the motivation for our study. Let $(\vec{z}, \vec{\theta})$ denote the initial positions and velocities of these particles, and $f_{i}((\vec{z}, \vec{\theta}))$ denote the number of particles pointing in the direction $i \in\{ \pm \theta, \pm(\pi-\theta)\}$. If the functions $f_{i}$ were integrable, then we could give a nice answer to this question using Theorem 3, but unfortunately this is not the case. We give three partial answers. First a finite measure version. Let $A \subset \mathcal{B}^{g}$ be a positive but finite measure subset of the wind-tree table, and let $f_{i}^{A}$ denote the function $f_{i}$ restricted to the set $A \times \cdots \times A$. This function is integrable, thus applying the Hopf ergodic theorem to the wind-tree flow yields the following corollary (here $K$ and $s$ are fixed, and $G$ and $\mathcal{H}$ are the dense $G_{\delta}$ sets from Theorem 3 )

Corollary 4. For each $g \in G$, for each $A \subset \mathcal{B}^{g}$ of positive measure, for each $\theta \in \mathcal{H}$, for each $i, j$ the following limit holds almost surely as 
$T \rightarrow \infty:$

$$
\frac{\int_{0}^{T} f_{i}^{A}\left(\phi_{t}^{\theta} \times \cdots \phi_{t}^{\theta}(\vec{z}, \vec{\theta})\right) d t}{\int_{0}^{T} f_{j}^{A}\left(\phi_{t}^{\theta} \times \cdots \phi_{t}^{\theta}(\vec{z}, \vec{\theta})\right) d t} \rightarrow 1 .
$$

This means that if we only count when all the particles are in the set $A$ then the average over times of the number going in each direction is asymptotically the same.

If we replace the flow $\phi_{t}^{\theta} \times \cdots \phi_{t}^{\theta}$ by its first return flow $\psi_{t}^{A, \vec{\theta}}$ to the region $A \times \cdots \times A$, then we can apply the Birkhoff ergodic theorem.

Corollary 5. For each $g \in G$, for each $A \subset \mathcal{B}^{g}$ of positive measure, for each $i$, the following limit holds almost surely as $T \rightarrow \infty$ :

$$
\frac{1}{T} \int_{0}^{T} f_{i}^{A}\left(\psi_{t}^{A, \vec{\theta}}(\vec{z}, \vec{\theta})\right) d t \rightarrow \int_{A} f_{i}^{A} d \mu \times \cdots \times d \mu=\frac{K}{4} \cdot \operatorname{area}(A) .
$$

This means that the average over time of the direction converges to $K / 4$, but for the first return flow.

Finally we can replace the $f_{i}$ by integrable functions which somehow measure a similar phenomenon. For example the sum of the cubes of the reciprocal of the distance of the particles from the origin: $\hat{f}_{i}((\vec{z}, \vec{\theta}))=\sum_{\left\{k: \vec{\theta}_{k}=i\right\}} \frac{1}{\min \left(1,\left|z_{k}\right|^{3}\right)}$. These functions are positive and integrable, thus we can apply the Hopf ergodic theorem to conclude:

Corollary 6. For each $g \in G$, for each $\theta \in \mathcal{H}$, for each $i, j$ the following limit holds almost surely as $T \rightarrow \infty$ :

$$
\frac{\int_{0}^{T} \hat{f}_{i}\left(\phi_{t}^{\theta} \times \cdots \phi_{t}^{\theta}(\vec{z}, \vec{\theta})\right) d t}{\int_{0}^{T} \hat{f}_{j}\left(\phi_{t}^{\theta} \times \cdots \phi_{t}^{\theta}(\vec{z}, \vec{\theta})\right) d t} \rightarrow 1 .
$$

This means that then the average over time of the weighed number going in each direction is asymptotically the same.

For all three results we can replace a single $\theta \in \mathcal{H}$ by a vector $\vec{\theta} \in \mathcal{H}(G)$ and state a similar result for the functions $f_{i}, f_{i}^{A}, \hat{f}_{i}$ which counts the number of particles with direction in the $i$ th quadrant $(i \in\{1,2,3,4\})$. We interpret these results in the following way, if $K$ particles are launched in arbitrary generic directions, then the average over time of the number of particles in the different quadrants are asymptotically the same in the three senses mentioned above.

\section{Proof of Wind-TREe RESUlts}

Fix a wind-tree configuration $g \in$ Conf. Fix $K$ and $n \in \mathbb{N}$, and let $\mathcal{B}_{n}^{g}:=\left(\mathcal{B}^{g} \cap\{(x, y):|x|+|y| \leq n s\}\right)^{K}$. Note that $K$ does not appear in this notation, as well as certain other notations in this section, since it is fixed throughout much of the proof. For each $\theta$ let $[\theta]$ be the set of all possible directions under the billiard flow starting in direction $\theta$, 

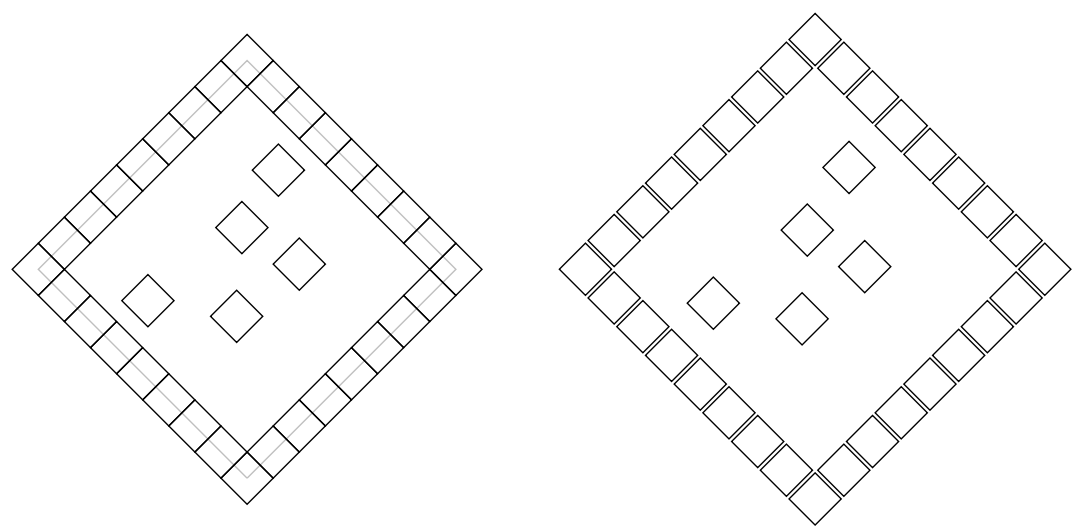

FiguRE 1. An 8-ringed configuration and a configuration close to it.

i.e., $[\theta]=\{ \pm \theta, \pm(\pi-\theta)\}$. Let $[\vec{\theta}]:=\left\{\vec{\psi}: \psi_{i} \in\left[\theta_{i}\right]\right.$ for all $\left.i\right\}$ and

$$
X_{n}^{g, \vec{\theta}}=\left\{(\vec{z}, \vec{\psi}): \vec{z} \in \mathcal{B}_{n}^{g}, \vec{\psi} \in[\vec{\theta}]\right\} .
$$

For each $n \geq 1$ we consider the first return flow of the product billiard flow,

$$
\phi_{t}^{g, \vec{\theta}, n}: X_{n}^{g, \vec{\theta}} \rightarrow X_{n}^{g, \vec{\theta}} .
$$

For each $\theta \in \mathbb{S}^{1}$, the flow $\phi_{t}^{g, \vec{\theta}, n}$ preserves the measure $\mu^{K}$. For sake of simplicity, we will denote $\mu^{K}$ by $\mu$.

Proof of Theorem 3. We prove both statements with the same strategy: we choose a dense set $\left\{f_{i}\right\}$ of configurations which satisfy the goal dynamical property of $K$-fold ergodicity on certain compact sets. Then we will show that wind-tree tables which are sufficiently well approximated by this dense set will satisfy the dynamical property on the whole phase space. The proof for $K=1$ is simpler, and we will mention the simplification in the proof even though this is not formally necessary for the proof.

A configuration $h$ is called $n$-ringed if the boundary of the rhombus $\left\{(x, y) \in \mathbb{R}^{2}:|x|+|y| \leq n s\right\}$ is completely covered by trees as in Figure 1 left (i.e., the obstacles covering the boundary intersect with each other on a whole side or do not intersect at all).

For the proof of simple ergodicity, let $\left\{f_{i}\right\}$ be a dense set of parameters such that each $f_{i}$ is an $n_{i}$-ringed configuration and $n_{i}$ is increasing with $i$. Then by [KeMaSm] the billiard flow is ergodic in almost every direction inside the ring. So, the return flow $\phi_{t}^{\vec{\theta}, n}$ is ergodic for all $n$ such that $1 \leq n \leq n_{i}$ and for almost every direction $\theta$ where $\vec{\theta}$ is the vector $(\theta)$.

Consider now the $K$-fold case. Let $g$ be any configuration and $\varepsilon>0$. Let $n>\varepsilon+\frac{1}{\varepsilon}+s$. Consider the $n$-ringed configuration $f$ which coincides with $g$ inside the the ball of radius $1 / \varepsilon$ and has no additional trees. We apply Theorem 1 of [MSTr3] to the table in the interior of the ring of 
the ringed configuration $f$ yielding a dense $G_{\delta}$ set $\Theta$ of full measure of directions, and a configuration that is $n$-ringed, and is $\varepsilon$-close to $f$ and $g$ such that the flow is weakly mixing inside the ring for all $\theta \in \Theta$.

Thus, we can find a dense set of configurations $\left\{f_{i}\right\}$ such that each $f_{i}$ is $n_{i}$-ringed and the flow is weakly mixing for all $\theta \in \Theta$ inside the ring. Again we suppose $n_{i}$ is increasing with $i$.

Fix $K \geq 1$. Suppose that $\delta_{i}$ are strictly positive numbers. Then the set

$$
\mathcal{G}_{K}:=\bigcap_{m=1}^{\infty} \bigcup_{i=m}^{\infty} \mathcal{U}_{\delta_{i}}\left(f_{i}\right)
$$

is a dense $G_{\delta}$ set. We will show that the $\delta_{i}$ can be chosen in such a way that all the configurations in $\mathcal{G}$ are $K$-fold ergodic for all $\vec{\theta} \in \Theta^{K}$. This $\Theta$ will be a $G_{\delta}$ set of full measure that has to be found in the proof. Taking intersection over $K$ will finish the proof, thus for sake of simplicity, we will fix $K$ from here on and drop it from the notations when convenient.

Let $\left\{h_{j}\right\}_{j \geq 1}$ be a countable dense collection of continuous functions in $L^{1}\left(\mathbb{R}^{2 K}, L e b\right)$. For any $\vec{\theta}$ and $g \in$ Conf we think of this as a collection in $\mathcal{L}^{1}\left(X_{n}^{g, \vec{\theta}}, \mu_{n}^{K}\right)$ in the same way as in the proof of Theorem 1 of [MSTr3].

Consider the Cesaro average

$$
S_{n, \ell}^{g} h_{j}(\vec{z}, \vec{\theta}):=\frac{1}{\ell} \int_{0}^{\ell} h_{j}\left(\phi_{t}^{g, \vec{\theta}, n}(\vec{z}, \vec{\theta})\right) d t .
$$

By the Birkhoff ergodic theorem, the flow $\phi^{g, \vec{\theta}, n}$ is ergodic for all $n$ and for almost every $\theta$ if and only if for all $n$ and for almost all $\theta$ we have $S_{n, \ell}^{g} h_{j}(\vec{z}, \vec{\theta}) \rightarrow \int_{X_{n}^{\theta}}\left(h_{j}^{\theta}(y)\right) d \mu(y)$ as $\ell$ goes to infinity for all $j \geq 1$.

Now fix $i$. The billiard flow $\phi_{t}^{f_{i}, \theta}$ is weakly-mixing inside the ring for each $\theta \in \Theta$, thus $\phi_{t}^{f_{i}, \vec{\theta}}$ inside the ring is ergodic for every $\vec{\theta}$ in $\Theta^{K}$. Thus the first return flows $\phi_{t}^{f_{i}, \vec{\theta}, n}$ are ergodic for every $\vec{\theta}$ in $\Theta^{K}$, for all $1 \leq n \leq n_{i}$. Thus we can find positive integers $\ell_{i} \geq n_{i}$, open sets $H_{i} \subset \mathbb{S}^{1}$ and sets $C_{n}^{f_{i}, \vec{\theta}} \subset X_{n}^{f_{i}, \vec{\theta}}$ so that $\mu\left(C_{n}^{f_{i}, \vec{\theta}}\right)>\mu\left(X_{n}^{f_{i}, \vec{\theta}}\right)-\frac{1}{i}$, $\lambda\left(H_{i}\right)>1-\frac{1}{i}$ and

$$
\left|S_{n, \ell_{i}}^{f_{i}} h_{j}(\vec{z}, \vec{\theta})-\int_{X_{n}^{f_{i}, \vec{\theta}}} h_{j}(y) d \mu(y)\right|<\frac{1}{i}
$$

for all $\vec{z} \in C_{n}^{f_{i}, \vec{\theta}}, \vec{\theta} \in\left(H_{i}\right)^{K}, 1 \leq j \leq i$, and $1 \leq n \leq n_{i}$.

Now we would like to extend these estimates to the neighborhood $\mathcal{U}_{\delta_{i}}\left(f_{i}\right)$ for a sufficiently small strictly positive $\delta_{i}$ (see Figure 1 right). For any $n$ such that $1 \leq n \leq n_{i}$ let $\bar{B}_{n}^{i}$ be the intersection of $\mathcal{B}_{n}^{g}$ for all $g$ in the $\delta_{i}$-neighbourhood $\mathcal{U}_{\delta_{i}}\left(f_{i}\right)$. Let $\bar{X}_{n}^{i, \vec{\theta}}:=\bar{B}_{n}^{i} \times[\vec{\theta}]$. For every $1 \leq n \leq n_{i}$ we define $\vec{\psi}=\vec{\psi}\left(g, f_{i}\right)$ a piecewise continuous map from $\mathcal{B}_{n}^{g}$ to $\mathcal{B}_{n}^{f_{i}}$. When convenient we will write $\vec{\psi}(\vec{z}, \vec{\theta})$ instead of $(\vec{\psi}(\vec{z}), \vec{\theta})$. The 
behavior of $\vec{\psi}$ will be defined coordinate by coordinate, more precisely $\vec{\psi}(\vec{z})=\left(\psi\left(z_{1}\right) \ldots, \psi\left(z_{K}\right)\right)$ where $\psi$ will be defined right now. For $z$ outside the obstacles of $f_{i}$, we define $\psi(z)=z$. For each obstacle $O_{1}$ of $g$ inside the ring, we consider the corresponding obstacle $\mathrm{O}_{2}$ of $f_{i}$ and $C_{12}=O_{2} \backslash O_{1}$. We define a direction $\xi$ that points from a corner of $O_{1}$ to a corner of $O_{2}$ in such a way that the segment along this direction between the two corners is completely included in $C_{12}$ (as in Figure 21). Then for any $z \in C_{12}$, the image $\psi(z)$ of $z$ is the closest point in the direction $\xi$ in the table $\mathcal{B}_{n}^{f_{i}}$ (Figure 2). The difference between the Lebesgue measure of $\bar{B}_{n}^{i}$ and the measure of $\mathcal{B}_{n}^{g}$ can be made arbitrarily small by an adequate choice of $\delta_{i}$, simultaneously for all $g$ in $\mathcal{U}_{\delta_{i}}\left(f_{i}\right)$. From now on, we make the choice of $\delta_{i}$ such that $\mu\left(\bar{X}_{n}^{i, \vec{\theta}}\right)=\mu\left(\bar{B}_{n}^{i}\right)>\mu\left(\mathcal{B}_{n}^{g}\right)-\frac{1}{i}$ for all $g \in \mathcal{U}_{\delta_{i}}\left(f_{i}\right)$ and all $i$.

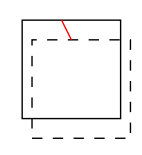

Figure 2. The dashed obstacle is $O_{1}$ and the solid obstacle is the associated obstacle $O_{2}$. The map $\psi$ maps all the points on the red segment in the direction $\xi$ to a point in its top endpoint.

By the triangular inequality we have:

$$
\begin{aligned}
& \left|S_{n, \ell_{i}}^{g} h_{j}(\vec{z}, \vec{\theta})-\int_{X_{n}^{g, \vec{\theta}}} h_{j}(y) d \mu(y)\right| \leq\left|S_{n, \ell_{i}}^{g} h_{j}(\vec{z}, \vec{\theta})-S_{n, \ell_{i}}^{f_{i}} h_{j}(\vec{\psi}(\vec{z}), \vec{\theta})\right|+ \\
& \left|S_{n, \ell_{i}}^{f_{i}} h_{j}(\vec{\psi}(\vec{z}), \vec{\theta})-\int_{X_{n}^{f_{i}, \vec{\theta}}}\left(h_{j}(y)\right) d \mu(y)\right|+ \\
& \left|\int_{X_{n}^{f_{i}, \vec{\theta}}} h_{j}(y) d \mu(y)-\int_{X_{n}^{g, \vec{\theta}}} h_{j}(y) d \mu(y)\right| \text {. }
\end{aligned}
$$

Futhermore we choose $\delta_{i}$ so small that

$$
\left|\int_{X_{n}^{f_{i}, \vec{\theta}} \backslash \bar{X}_{n}^{i, \vec{\theta}}} h_{j}(y) d \mu(y)\right|<\frac{1}{i}
$$

and

thus by the triangular inequality

$$
\left|\int_{X_{n}^{g, \vec{\theta}} \backslash \bar{X}_{n}^{i, \vec{\theta}}} h_{j}(y) d \mu(y)\right|<\frac{1}{i}
$$

$$
\left|\int_{X_{n}^{f_{i}, \vec{\theta}}} h_{j}(y) d \mu(y)-\int_{X_{n}^{g, \vec{\theta}}} h_{j}(y) d \mu(y)\right|<\frac{2}{i} .
$$

Now the proof bifurcates a bit according to the different cases stated in the theorem. Consider part (1) of the theorem. So in particular $\vec{\theta}$ will indicate $(\theta, \theta, \ldots, \theta)$ in this part. Note that $\psi$ is not continuous, not invertible, and not onto. However it is not far from being continuous: $\|\vec{z}-\vec{\psi}(\vec{z})\|_{\mathcal{L}^{\infty}}<\delta_{i}$ for any $\vec{z} \in \mathcal{B}_{n}^{g}$. By our convention the billiard flow stops at corners, thus any point $(\vec{z}, \vec{\theta})$ for which the flow is defined up 
to time $\ell_{i}$ is a point of continuity for $\phi_{\ell_{i}}^{f_{i}, \vec{\theta}, n}$. Consider such a point, then the point $\vec{\psi}\left(\phi_{\ell_{i}}^{g, \vec{\theta}, n}(\vec{z}, \vec{\theta})\right)$ stays $\delta_{i}$-close to $\phi_{\ell_{i}}^{f_{i}, \vec{\theta}, n}(\vec{z}, \vec{\theta})$ for $g$ in a small enough neighborhood of $f_{i}$; thus we can find $\delta_{i}>0$, an open set $\hat{H}_{i} \subset H_{i}$ and a set $\hat{C}_{n}^{i, \vec{\theta}} \subset \bar{X}_{n}^{i, \vec{\theta}} \cap C_{n}^{f_{i}, \vec{\theta}}$ so that that if $g \in \mathcal{U}\left(f_{i}, \delta_{i}\right)$, then

$$
\left|S_{n, \ell_{i}}^{g} h_{j}(\vec{z}, \vec{\theta})-S_{n, \ell_{i}}^{f_{i}} h_{j}(\vec{\psi}(\vec{z}), \vec{\theta})\right|<\frac{2}{i}
$$

for all $z \in \hat{C}_{n}^{i, \vec{\theta}}, \theta \in \hat{H}_{i}$ (here $\left.\vec{\theta}=(\theta, \theta, \ldots, \theta)\right), 1 \leq j \leq i, 1 \leq n \leq n_{i}$; and $\mu\left(\hat{C}_{n}^{i, \vec{\theta}}\right)>\mu\left(\mathcal{B}_{n}^{g}\right)-\frac{2}{i}$ and $\hat{H}_{i}$ is of measure larger than $1-\frac{2}{i}$.

Since $\lambda\left(\hat{H}_{i}\right)>1-2 / i$, the set $\mathcal{H}=\cap_{M=1}^{\infty} \cup_{i=M}^{\infty} \hat{H}_{i}$ has full measure. Fix $g \in \mathcal{G}$ and $\theta \in \mathcal{H}$, then there is an infinite sequence $i_{k}$ such that $g \in$ $\mathcal{U}_{\delta_{i_{k}}}\left(f_{i_{k}}\right)$ and $\theta \in \hat{H}_{i_{k}}$. Fix $n \geq 1$ and consider $\mathcal{C}_{n}^{g, \vec{\theta}}:=\cap_{M=1}^{\infty} \cup_{k=M}^{\infty} \widehat{C}_{n}^{i_{k}, \vec{\theta}}$. Recall that we made the choice of $\delta_{i}$ such that $\mu\left(\bar{X}_{n}^{i_{k}, \vec{\theta}}\right)>\mu\left(\mathcal{B}_{n}^{g}\right)-\frac{1}{i_{k}}$. Since $\mu\left(\widehat{C}_{n}^{i_{k}, \vec{\theta}}\right)>\mu\left(\bar{X}_{n}^{i_{k}, \vec{\theta}}\right)-\frac{1}{i_{k}}$, it follows that $\mu\left(\mathcal{C}_{n}^{g, \vec{\theta}}\right)=\mu\left(\mathcal{B}_{n}^{g}\right)$.

Suppose $g \in \mathcal{G}$. Thus for $\theta \in \mathcal{H}$, for each $n \geq 1$ the three inequalities (1), (2), (3) imply that

$$
\left|S_{n, \ell_{i_{k}}}^{g}\left(h_{j}^{\theta}\right)-\int_{X_{n}^{\theta}}\left(h_{j}^{\theta}(\vec{z}, \vec{\theta})\right) d \mu\right|<\frac{5}{i}
$$

for all $z \in \hat{C}_{n}^{i, \vec{\theta}}, \theta \in \hat{H}_{i}$ (here $\left.\vec{\theta}=(\theta, \theta, \ldots, \theta)\right), 1 \leq j \leq i, 1 \leq n \leq n_{i}$ and thus

$$
\lim _{k \rightarrow \infty} S_{n, \ell_{i_{k}}}^{g}\left(h_{j}^{\theta}\right) \rightarrow \int_{X_{n}^{\theta}}\left(h_{j}^{\theta}(\vec{z}, \vec{\theta})\right) d \mu
$$

for all $(\vec{z}, \vec{\theta})$ in $\mathcal{C}_{n}^{g, \vec{\theta}}$, for each $j \geq 1$. The $h_{j}^{\theta}$ are dense in $L^{1}\left(X_{n}^{\theta}, \mu\right)$ and $\lim _{k \rightarrow \infty} \ell_{i_{k}}=\infty$, thus Equation (44) together with the Birkhoff ergodic theorem imply that for each $n \geq 1$, the first return flow $\phi_{t}^{g, \vec{\theta}, n}$ is ergodic for all $\theta \in \mathcal{H}$. This implies the ergodicity of the billiard flow $\phi_{t}^{g, \vec{\theta}}$ in every direction in $\mathcal{H}$.

For part (2) of the theorem we have to slightly modify the previous arguments. The only difference being that the set of directions we construct depends on $K$. For any point $(\vec{z}, \vec{\theta})$ of continuity of $\phi_{\ell_{i}}^{f_{i}, \vec{\theta}, n}$, the point $\phi_{\ell_{i}}^{g, \vec{\theta}, n}(\vec{z}, \vec{\theta})$ varies continuously with $g$ in a small neighborhood of $f_{i}$; thus we can find $\delta_{i}>0$, an open set $\hat{H}_{i}(K) \subset\left(H_{i}\right)^{K}$ and a set $\hat{C}_{n}^{i, \vec{\theta}} \subset \bar{X}_{n}^{i, \vec{\theta}} \cap C_{n}^{f_{i}, \vec{\theta}}$ so that that if $g \in \mathcal{U}\left(f_{i}, \delta_{i}\right)$, then

$$
\left|S_{n, \ell_{i}}^{g} h_{j}(\vec{z}, \vec{\theta})-S_{n, \ell_{i}}^{f_{i}} h_{j}(\vec{\psi}(\vec{z}), \vec{\theta})\right|<\frac{2}{i}
$$

for all $z \in \hat{C}_{n}^{i, \vec{\theta}}, \vec{\theta} \in \hat{H}_{i}(K), 1 \leq n \leq n_{i}, 1 \leq j \leq i$; and $\mu\left(\hat{C}_{n}^{i, \vec{\theta}}\right)>$ $\mu\left(\mathcal{B}_{n}^{g}\right)-\frac{2}{i}$ and $\hat{H}_{i}(K)$ is of measure larger than $1-\frac{2}{i}$.

Since $\lambda\left(\hat{H}_{i}\right)>1-2 / i$, the $G_{\delta}$ set $\mathcal{H}(K)=\cap_{M=1}^{\infty} \cup_{i=M}^{\infty} \hat{H}_{i}(K)$ has full measure. The rest of the proof of part (2) is identical to that of 
part (1).

3.1. Generalization. If we consider a subset $C$ of $\left(\right.$ Conf, $\left.d_{H}\right)$ which is itself a Baire set such that the set $\left\{h: h\right.$ is $\mathrm{N}$-ringed for $\left.N \geq N_{0}\right\}$ is dense in $C$ for each $N_{0} \geq 1$ then Theorem 3 holds in $\left(C, d_{H}\right)$ as well. In particular the set of configurations considered in the articles MSTr1, MSTr2] is a Baire subset of $\left(\right.$ Conf, $\left.d_{H}\right)$ thus Theorem 3 holds in that context as well.

\section{APPENDiX}

Proof of Proposition 1. Let $\left(g_{i}\right)_{i \in \mathbb{N}}$ be a sequence of configurations. Consider $\varepsilon_{n}=1 / n$. Let $\left.B_{\varepsilon}:=\{z: \rho(z, \infty)>\varepsilon)\right\}$. Let $k_{j}$ be the cardinality of $g_{j} \cap B_{\varepsilon_{1}}$. The sequence $k_{i}$ only take a finite number of values. Thus we can choose subsequence $\left(g_{j}\right)_{j \in J_{0}}$ such that the sequence $\left(k_{j}: j \in J_{0}\right)$ is constant, call this constant $c_{1}$.

If $c_{1}=0$ then for each $j \in J_{0}$ let $g_{j}^{1}$ be the empty configuration. Otherwise for each $j \in J_{0}$ let $g_{j}^{1}:=\left\{z_{1, j}^{1}, \ldots, z_{c_{1}, j}^{1}\right\}=g_{j} \cap B_{\varepsilon_{1}}$. For each $j$ we think of $g_{j}^{1}$ as a finite configuration in Conf, but also as a point in $\mathbb{S}^{c_{1}}$. By compactness of $\mathbb{S}^{c_{1}}$ we can find a subsequence $J_{1} \subset J_{0}$ such that the $\left(g_{j}^{1}: j \in J_{1}\right)$ converge to a point $g^{1}:=\left(z_{1}^{1}, \ldots, z_{c_{1}}^{1}\right) \in \mathbb{S}^{c_{1}}$. Note that $d\left(z_{i}^{1}, z_{j}^{1}\right) \geq s$ for all $i \neq j$, thus $g^{1} \in$ Conf. Furthermore we have $g_{j}^{1} \in \mathcal{U}_{\varepsilon_{1}}\left(g^{1}\right)$ for all sufficiently large $j \in J_{1}$. Repeat this argument for $n=2$ to produce a subsequence $J_{2} \subset J_{1}$ which converge to a point $g^{2} \in \mathbb{S}^{c_{2}}$. Again we have $g^{2} \in$ Conf and $g_{j}^{2} \in \mathcal{U}_{\varepsilon_{2}}\left(g^{2}\right)$ for all sufficiently large $j \in J_{2}$. Note that $c_{2} \geq c_{1} \geq 0$ and for $j=1, \ldots, c_{1}$ we have $z_{j}^{2}=z_{j}^{1}$. Repeat this construction for each $n$. Finally we define $g$ to be an almost countable collection of points such that every $z \in g$ is in $g^{k}$ for all sufficiently large $k$. By construction for any $z_{1}, z_{2} \in g$ we have $d\left(z_{1}, z_{2}\right) \geq s$, thus $g \in$ Conf. Note that $g$ can be an infinite, finite, or even the empty configuration.

We claim that $g$ is an accumulation point of the sequence $\left(g_{j}\right)_{j \in \mathbb{N}}$. Fix a neighborhood $\mathcal{U}$ of $g$. Choose $n$ so large that $\mathcal{U}_{\varepsilon_{n}}(g) \subset \mathcal{U}$. By construction of $g$ and $g^{n}$ we have $\mathcal{U}_{\varepsilon_{n}}\left(g^{n}\right)=\mathcal{U}_{\varepsilon_{n}}(g)$. The result follows since $g_{j} \in \mathcal{U}_{\varepsilon_{n}}\left(g^{n}\right)$ for all sufficiently large $j \in J_{n} \subset \mathbb{N}$.

Remark. If we remove the empty and finite configurations from Conf the space is not even locally compact. 


\section{REFERENCES}

[AvHu] A. Avila and P. Hubert, Recurrence for the wind-tree model Annales de l'Institut Henri Poincaré - Analyse non linéaire.

[BaKhMaPl] P. Bachurin, K. Khanin, J. Marklof and A. Plakhov Perfect retroreflectors and billiard dynamics Journal of Modern Dynamics 5 (2011) 33-48.

[BiRo] C. Bianca and L. Rondoni The nonequilibrium Ehrenfest gas: A chaotic model with flat obstacles? Chaos 19 (2009) 013121.

[De] V. Delecroix Divergent trajectories in the periodic wind-tree model J. Mod. Dyn. 7 (2013) 1-29.

[DeHuLe] V. Delecroix, P. Hubert and S. Lelièvre Diffusion for the periodic windtree model Ann. Sci. ENS 47 (2014) 1085-1110.

[DeZo] V. Delecroix and A. Zorich Cries and whispers in wind-tree forests arXiv:1502.06405 (2015)

[DeCoVB] C.P. Dettmann, E.G.D. Cohen and H. van Beijeren Statistical mechanics: Microscopic chaos from brownian motion? Nature 401, 875 (1999) doi:10.1038/44759

[EhEh] P. and T. Ehrenfest Begriffliche Grundlagen der statistischen Auffassung in der Mechanik Encykl. d. Math. Wissensch. IV 2 II, Heft 6, 90 S (1912) (in German, translated in:) The conceptual foundations of the statistical approach in mechanics, (trans. Moravicsik, M. J.), 10-13 Cornell University Press, Itacha NY (1959).

[FrHu] K. Fracczek and P. Hubert Recurrence and non-ergodicity in generalized wind-tree models arXiv:1506.05884 (2015).

[FrUl] K. Frączek and C. Ulcigrai Non-ergodic $\mathbb{Z}$-periodic billiards and infinite translation surfaces Invent. Math. 197 (2014) 241-298.

[Ga] G. Gallavotti Divergences and the Approach to Equilibrium in the Lorentz and the Wind-Tree Models Phys. Rev. 185 (1969) 308-322.

[GaBoGe] G. Gallavotti, F. Bonetto and G. Gentile, Asepcts of Ergodic Qualitative and Statistical Theory of Motion Springer 2004

[HaWe] J. Hardy and J. Weber Diffusion in a periodic wind-tree model J. Math. Phys. 21 (1980) 1802-1808.

[HaCo] E.H. Hauge and E.G.D. Cohen Normal and Abnormal Diffusion in Ehrenfest's Wind-Tree Model J. Math. Phys. 10 (1969) 397-414.

[HaCo1] E.H. Hauge and E.G.D. Cohen Normal and Abnormal Diffusion in Ehrenfest's Wind-Tree Model Phys. Lett. A 25 (1967) 78-79.

[HuLeTr] P. Hubert, Pascal, S. Lelièvre and S. Troubetzkoy The Ehrenfest windtree model: periodic directions, recurrence, diffusion J. Reine Angew. Math. 656 (2011) 223-244.

[KeMaSm] S. Kerckhoff, H. Masur, and J. Smillie, Ergodicity of billiard flows and quadratic differentials, Annals of Math. (2) 124 (1986), no. 2, 293-311.

[MSTr1] A. Málaga Sabogal and S. Troubetzkoy Minimality of the Ehrenfest windtree model Journal Modern Dynamics 10 (2016), 209-228.

[MSTr2] A. Málaga Sabogal and S. Troubetzkoy Ergodicity of the Ehrenfest windtree model Comptes Rendus Mathematique 354 (2016) 1032-1036.

[MSTr3] A. Málaga Sabogal and S. Troubetzkoy Weakly-mixing polygonal billiards Bulletin London Math. Soc. (to appear).

[Tr] S. Troubetzkoy Typical recurrence for the Ehrenfest wind-tree model J. Stat. Phys. 141 (2010) 60-67.

[VBHa] H. Van Beyeren and E.H. Hauge, Abnormal diffusion in Ehrenfest's windtree model Physics Letters A 39, (1972) 397-398.

[WoLa] W. Wood and F. Lado Monte Carlo calculation of normal and abnormal diffusion in Ehrenfest's wind-tree model J. Comp. Physics 7 (1971) 528-546. 
CHArt/THiM (EA 4004) Université PARis 8-Vincennes-SAint-Denis.

E-mail address: alba.malaga@polytechnique.edu

Aix Marseille Univ, CNRS, Centrale Marseille, I2M, Marseille, FRANCE

Address: I2M, Luminy, Case 907, F-13288 Marseille CEDEX 9, France

E-mail address: serge.troubetzkoy@univ-amu.fr 